Aim of the study: Our objective was to quantify the accuracy of dose calculation in the build-up region of the tangential field of the breast for a TiGRT treatment planning system (TPS) Material and methods: Thermoluminescent dosimeter (TLD) chips were arranged in a RANDO phantom for the dose measurement. TiGRT TPS was also used for the dose calculation. Finally, confidence limit values were obtained to quantify the accuracy of the dose calculation of the TPS at the build-up region.

Results: In the open field, for gantry angles of $15^{\circ}, 30^{\circ}$, and $60^{\circ}$, the confidence limit values were $17.68,19.97$, and 34.62 at a depth of $5 \mathrm{~mm}$, and 24.01, 19.07, and 15.74 at a depth of $15 \mathrm{~mm}$, respectively. In the wedge field, for gantry angles of $15^{\circ}, 30^{\circ}$, and $60^{\circ}$, the confidence limit values were $21.64,26.80$, and 34.87 at a depth of $5 \mathrm{~mm}$, and 27.92, 22.04, and 20.03 at a depth of $15 \mathrm{~mm}$, respectively. Additionally, the findings showed that at a depth of $5 \mathrm{~mm}$, the confidence limit values increased with increasing gantry angle while at a depth of $15 \mathrm{~mm}$, the confidence limit values decreased with increasing gantry angle.

Conclusions: Overall, TiGRT TPS overestimated doses compared to TLD measurements, and the confidence limit values were greater for the wedge field than for the open fields. Our findings suggest that the assessment of dose distributions in largedose gradient regions (i.e. build-up region) should not entirely rely on TPS calculations.

Key words: build up region,TPS, dose calculation accuracy, radiotherapy, TiGRT, surface dose.

Contemp Oncol (Pozn) 2017; 21 (3): 232-239 DOI:https://doi.org/10.5114/wo.2017.70114

\section{Assessment of the accuracy of dose calculation in the build-up region of the tangential field of the breast for a radiotherapy treatment planning system}

\author{
Bagher Farhood ${ }^{1}$, Mohammad Taghi Bahreyni Toossi², \\ Shokouhozaman Soleymanifard ${ }^{2}$, Tohid Mortezazadeh ${ }^{1}$
}

${ }^{1}$ Medical Physics and Medical Engineering Department, Faculty of Medicine, Tehran University of Medical Sciences, Tehran, Iran

${ }^{2}$ Medical Physics Research Center, Mashhad University of Medical Sciences, Mashhad, Iran

\section{Introduction}

Breast cancer can be treated using surgery, radiotherapy, chemotherapy, and hormone therapy [1]. Radiotherapy plays a significant role in the multimodal treatment of breast cancer. It has been shown in several studies to eventually improve survival and reduce locoregional recurrence [2-5]. There are many treatment techniques of radiotherapy for breast cancer, and one of them is two tangential wedged fields [6-12]. For megavoltage photon beams, the maximum dose is obtained at a relatively large depth [13]. However, the dose received by build-up regions or those close to the skin may have a paramount role in tumour recurrence prevention, particularly in cases in which the target volume has extended near the skin [14], making it necessary to consider doses close to the skin or build-up regions [13]. Additionally, the surgical scar should be covered by the prescribed dose to ensure the removal of seeded malignant cells and decrease the probability of tumour recurrence in the chest wall after mastectomy as well as around the surgical scar in breast conservation therapy [15]. On the other hand, adverse skin reactions caused by radiation therapy can be a limiting factor for breast cancer treatment [16]. Hence, an accurate calculation of the dose distribution is significant to ensure dose coverage of the target volume and prevent skin complications [17].

For the megavoltage photon beams used in radiotherapy, dose distribution in the surface/build-up region usually depends on field size, beam spectrum, angle of beam incidence, electron contamination, bolus or beam spoiler, source to skin distance, treatment techniques, and wedge [18-24]. In most cases, modern radiotherapy treatment planning systems (TPSs) are capable of accurately calculating doses in patients; however, surface and near-surface doses calculated by TPSs are inaccurate [16]. Therefore, the accurate calculation of dose distribution in the large-dose gradient region like the build-up region remains a challenge for most of the available photon dose calculation algorithms. This is due to difficulties in modelling the dose contributions from contaminated electrons derived from the flattening filter, collimator assembly, and secondary scatter photons from the accelerator head [25-29].

There are several studies relevant to dose calculation accuracy in the surface/build-up region of the breast for different algorithms and TPSs in radiotherapy [14, 15, 17, 30,31]. Akino et al. [15] evaluated the accuracy of superficial dose calculation using Eclipse TPS (Varian Medical Systems, Palo 
Alto, CA, USA) for several breast cancer treatment techniques. They concluded that TPS even with an advanced algorithm does not accurately predict the dose value in the build-up region. Moreover, Chow et al. [30] evaluated the calculation accuracy of superposition/convolution algorithms for tangential photon beams. They stated that the collapsed cone convolution and anisotropic analytical algorithm (AAA) cannot accurately predict dose distribution in depths $<2 \mathrm{~mm}$. Polednik et al. [31] evaluated the accuracy of dose calculation algorithms of various planning systems for tangential radiotherapy in breast cancer and reported that the collapsed cone calculates the dose distribution more accurately than the pencil beam convolution (PBC). In addition, Panettieri et al. [14] evaluated PBC and AAA calculation accuracy in the surface build-up region for tangential beam treatments and reported that the absorbed dose of the $6 \mathrm{MV}$ photon beam in the surface build-up region might change considerably depending on the type of algorithm used.

To the best of our knowledge, the dose calculation accuracy in the surface/build-up region of the tangential field of the breast for TiGRT TPS (LinaTech, Sunnyvale, CA, USA) has not yet been investigated. In the present study, we investigated the accuracy of the dose calculation in the build-up region of the tangential field of the breast for TiGRT TPS using a thermoluminescent dosimeter (TLD). Furthermore, we evaluated the effects of various gantry angles and the wedge on TPS dose calculation accuracy.

\section{Material and methods}

\section{Treatment planning and phantom irradiation}

A computed tomography (CT) scan was taken of the breast region of a RANDO phantom (The Phantom Laboratory, Salem, NY, USA) to produce a standard treatment plan as well as make dose calculations at different points. The CT system (SOMATOM Sensation 16, Siemens AG, Forchheim, Germany) has 16 slices and the slice thickness was $0.5 \mathrm{~cm}$. The images were then exported to the TPS, a TiGRT version 1.2. The TiGRT TPS uses a three-dimensional (3-D) photon dose calculation algorithm based on full scatter convolution (FSC). According to the manufacturer, the TiGRT TPS uses an exclusive algorithm that was developed to facilitate accurate and fast calculations. The FSC algorithm applies basic beam data collected during commissioning including the beam profile, tissue maximum ratio, collimator parameters, and total scatter factors. The dose calculation time per beam for conventional and 3-D conformal techniques is $<10$ seconds. The algorithm separates the absorbed dose $(D)$ in a given point into the primary dose $\left(D_{p}\right)$ and the scatter dose $\left(D_{s}\right)$ :

$$
\mathrm{D}=D_{\mathrm{p}}+\mathrm{D}_{\mathrm{s}}(1)
$$

The primary dose $D_{p}(\vec{r})$ is obtained based on the convolution algorithm using the following formula:

$$
D_{p}(\vec{r})=\iiint \phi_{p}\left(\vec{r}^{\prime}\right) k_{p}\left(\vec{r}-\vec{r}^{\prime}\right) d V^{\prime}(2)
$$

where $\phi_{p}\left(\vec{r}^{\prime}\right)$ is the photon fluence at the surface of a ray passing through the surface to point $\vec{r}^{\prime}$, and $k_{p}(\vec{r}-\vec{r})$ denotes the electron transport kernel, explaining the dose distribution around the primary interaction site of the photon. This demonstrates that the electron transport modelling by this algorithm has been considered and the electron dose deposition kernel can be scaled for heterogeneities such as the lung, bone, and air cavities. Finally, $V$ 'states the differential calculation volume at point $\vec{r}$. The scatter dose $D_{s}(\vec{r})$ is derived from the following convolution equation:

$$
D_{s}(\vec{r})=\iiint \phi_{p}\left(\vec{r}^{\prime}\right) k_{s}(\vec{r}-\vec{r}) d V^{\prime}(3)
$$

In the FSC algorithm, multiple photon scattering is discarded and $k_{s}(\vec{r}-\vec{r})$ is the first scatter fluence kernel. This kernel can be derived from the electron transport kernel.

In this study, the breast region of the RANDO phantom was selected to evaluate the accuracy of dose calculations in the surface/build-up region using the TiGRT TPS. Tangential fields with and without wedge filters were planned, and the dose accuracy assessment was performed once in each manner. The wedge angle was $45^{\circ}$ and gantry angles were $15^{\circ}, 30^{\circ}$, and $60^{\circ}$. To evaluate the effect of various gantry angles on TPS dose calculation accuracy, measurements and calculations were performed separately for each of the angles. Our reason to choose these gantry angles was that it could almost cover the range of angles used to treat breast cancer with tangential fields; as in a previous study [32], these gantry angles were applied to assess the effect of gantry angle on TPS dose calculation accuracy. The source axis distance technique was applied to deliver a 200 cGy dose to the selected point (a point in slice 18 of the RANDO phantom). In the RANDO phantom, the planned fields covered slices 15-22. Figure 1 shows the therapeutic region on the RANDO phantom. Slices 16, 18 , and 20 of the RANDO phantom were investigated. Because of the limitation related to TLD chip size (TLD size, $3 \mathrm{~mm}$ ) for evaluating surface/build-up region, dose calculation accuracy was investigated for each slice at depths of 5 and $15 \mathrm{~mm}$ (i.e. effective measurement points at depths of 5 and $15 \mathrm{~mm}$ ). Ten points were investigated in each slice (five at $5 \mathrm{~mm}$ and five at $15 \mathrm{~mm}$ ). Figure 2 shows the arrangement of the TLD chips at depths of 5 and $15 \mathrm{~mm}$ in slice 18 of the RANDO phantom.

Before irradiation, the TLD chips were placed in appropriate points on the RANDO phantom. The RANDO phantom was then irradiated based on the treatment plan with 6-MV X-rays emitted from a Varian Clinac 600 accelerator (Varian, Palo Alto, CA, USA). The doses received by TLD chips were measured and compared to those calculated by the TPS.

\section{Calibration of applied dosimeters and dosimetric method}

The TLD readout and analysis were performed at the national medical physics research centre, which has a special TLD analysis protocol. TLD-100s manufactured by Harshaw Company (Bicron, NE, OR Solon, OH, USA) (made 


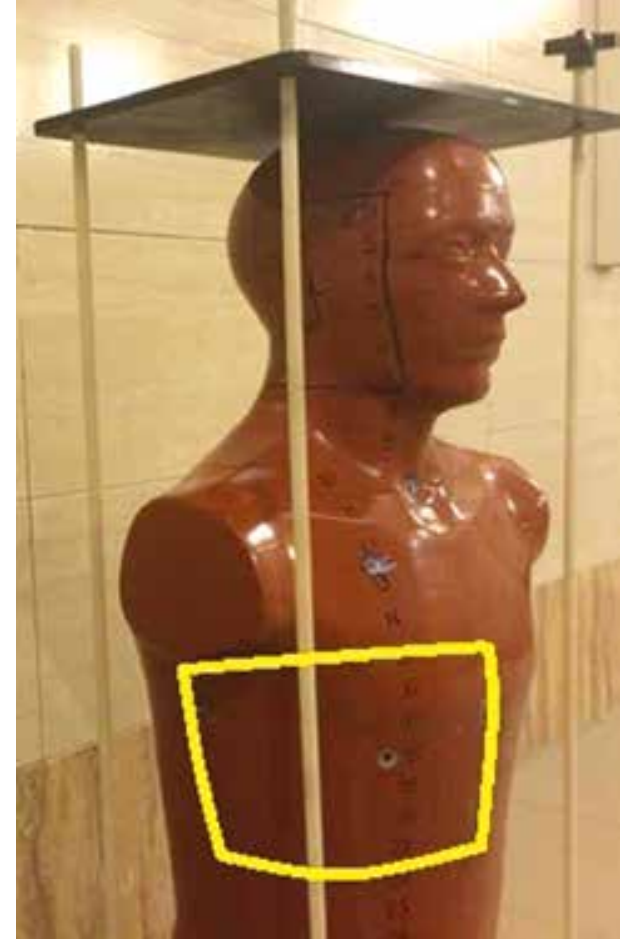

Fig. 1. Therapeutic range for the breast region on the RANDO phantom

of LiF, Mg, and Ti with a size of $3 \times 3 \mathrm{~mm}^{2}$ and thickness of $0.9 \mathrm{~mm}$ ) were used in this study. These TLDs have a reproducibility of approximately $\pm 1.5 \%$ ( $1 \mathrm{SD}$ ). Fifteen TLD chips were placed in a Perspex holder located on a $30-\mathrm{cm}$ water equivalent phantom. A $1.5-\mathrm{cm}$ water equivalent slab was placed on the holder to create a build-up region. Each was irradiated to determine their individual efficiency correction coefficient (ECC) and then irradiated with 50 cGy and read by a Harshaw reader to determine the reader calibration factor. Finally, the TLD chips were irradiated with $50 \mathrm{cGy}$ and their individual ECC were determined. A total of 180 dosimeters were placed in different areas of slices 16,18 , and 20 of the RANDO phantom. Four dosimeters were used to measure the background radiation. To increase the repeatability of the dosimetry results, the irradiations were repeated three times.

\section{Analysis of results}

The TRS 430 [33] and TECDOC 1540 [34] protocols were used to analyse the results. These protocols include information about TPS quality assurance. The difference between the measured and calculated dose is defined as:

$$
[\%]=100 \times\left(D_{\text {calc }}-D_{\text {meas }}\right) / D_{\text {meas }}(1) \delta
$$

where $D_{\text {calc }}$ and $D_{\text {meas }}$ are the doses calculated by the TPS and measured by the TLD-100, respectively. Therefore, the confidence limit is defined as:

$$
\Delta=\mid \text { average deviation } \mid+1.5 \times \operatorname{SD}(2)
$$

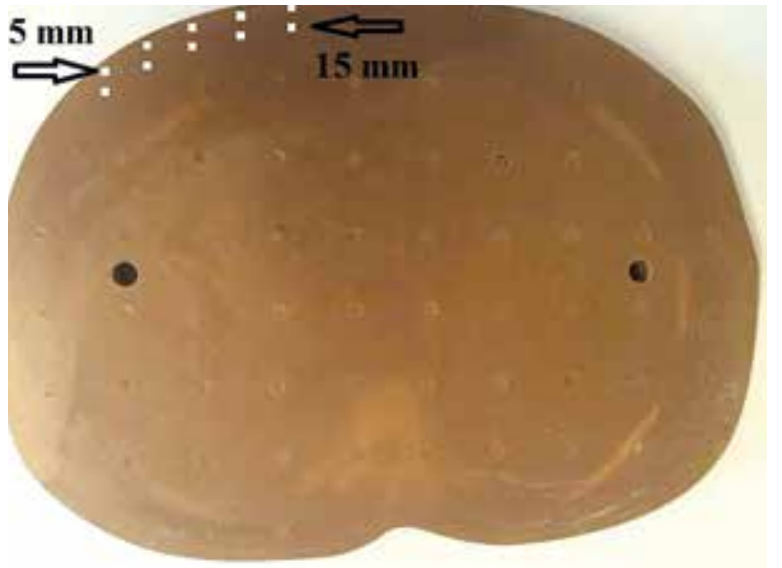

Fig. 2. Slice no. 18 of the RANDO phantom and arrangement of the thermoluminescent dosimeter chips at depths of $5 \mathrm{~mm}$ and $15 \mathrm{~mm}$

The confidence limit value is obtained by calculating the mean deviation between the calculated and measured dose values for several data points in comparable positions and the standard deviation of the differences (1 SD of the average).

Finally, the confidence limit values for each gantry angle in the wedged and non-wedged geometries were obtained and compared to the tolerance limit values suggested in the TRS 430 and TECDOC 1540 protocols. It is notable that the obtained confidence limit value for each gantry angle in the open and wedge fields includes 15 points ( 5 for each slice at each depth).

\section{Results}

In this study, dose values were measured in selected points inside the RANDO phantom for open and wedged fields in gantry angles of $15^{\circ}, 30^{\circ}$, and $60^{\circ}$. Next, dose values were calculated in the same selected points using the TiGRT TPS. Finally, differences between the measured and calculated doses were obtained, as these results are shown in Tables 1 and 2. Negative numbers in these tables reflect dose underestimation by the TPS, while positive numbers reflect dose overestimation by the TPS. Furthermore, for better understanding, the results are also shown in histograms (Fig. 3 and 4).

The dose differences (\%) between the TPS-calculated and TLD-measured doses of the open field with various gantry angles at depths of 5 and $15 \mathrm{~mm}$ are listed in Table 1. Figure 3 illustrates the dose differences (\%) between calculated and measured doses for open field with various gantry angles in depths of $5 \mathrm{~mm}$ (part a) and $15 \mathrm{~mm}$ (part b), respectively.

The dose differences (\%) between the TPS-calculated and TLD-measured doses for wedge field with various gantry angles at depths of 5 and $15 \mathrm{~mm}$ are listed in Table 2. Figure 4 illustrates the dose differences (\%) between calculations and measurements of the wedge field with various gantry angles at depths of 5 (part A) and $15 \mathrm{~mm}$ (part B), respectively. 
Table 1. Differences (\%) between the doses calculated by the TiGRT treatment planning system and those measured by the TLD-100 for the open field with gantry angles of $15^{\circ}, 30^{\circ}$, and $60^{\circ}$ at depths of $5 \mathrm{~mm}$ and $15 \mathrm{~mm}$

\begin{tabular}{|c|c|c|c|c|c|c|c|}
\hline \multirow{2}{*}{\multicolumn{2}{|c|}{$\frac{\text { Gantry angle }}{\text { Depth }}$}} & \multicolumn{2}{|c|}{$15^{\circ}$} & \multicolumn{2}{|c|}{$30^{\circ}$} & \multicolumn{2}{|c|}{$60^{\circ}$} \\
\hline & & $5 \mathrm{~mm}$ & $15 \mathrm{~mm}$ & $5 \mathrm{~mm}$ & $15 \mathrm{~mm}$ & $5 \mathrm{~mm}$ & $15 \mathrm{~mm}$ \\
\hline \multirow{15}{*}{ 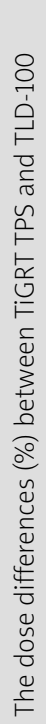 } & \multirow{5}{*}{ 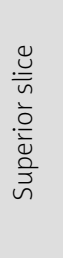 } & -17.38 & 0.84 & -11.85 & 9.1 & 10.2 & 12.17 \\
\hline & & 4.32 & -10.01 & 6.18 & 4.91 & 6.44 & 14.08 \\
\hline & & 8.97 & 3.09 & 5.35 & -2.19 & 25.22 & 5.86 \\
\hline & & 2.02 & 15.65 & 8.2 & 12.45 & 42.16 & 10.85 \\
\hline & & -14.08 & -10.4 & -1.13 & -8.99 & 25.68 & 13.78 \\
\hline & \multirow{5}{*}{ 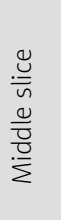 } & 6.86 & 10.52 & 6.67 & 6.62 & 10.95 & 7.84 \\
\hline & & -5.96 & 12.67 & -8.04 & 8.29 & 14.45 & 8.59 \\
\hline & & 5.07 & 15.28 & -0.34 & 15.46 & 19.67 & 16.06 \\
\hline & & 9.97 & -2.84 & 13.22 & -0.33 & 31.92 & 13.86 \\
\hline & & 16.87 & 14.31 & 6.6 & 13.54 & 32.08 & 13.02 \\
\hline & \multirow{5}{*}{ 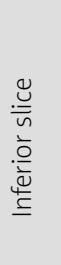 } & 15.15 & 13.9 & 25 & -7.66 & 6.92 & 9.69 \\
\hline & & -14.89 & 11.56 & -0.7 & -5.63 & 16.74 & 9.84 \\
\hline & & -4.22 & 17.47 & 14.32 & 15.51 & 13.45 & 3.66 \\
\hline & & 6.41 & 14.15 & 16.54 & 12.71 & 12.66 & 9.33 \\
\hline & & 5.73 & 24.06 & -8.39 & 15.07 & 6.62 & 11.86 \\
\hline
\end{tabular}

Table 2. Dose differences (\%) between the doses calculated by the TiGRT treatment planning system and the doses measured by the TLD-100 for the wedge field with gantry angles of $15^{\circ}, 30^{\circ}$, and $60^{\circ}$ at depths of $5 \mathrm{~mm}$ and $15 \mathrm{~mm}$

\begin{tabular}{|c|c|c|c|c|c|c|c|}
\hline \multirow{2}{*}{\multicolumn{2}{|c|}{$\begin{array}{c}\text { Gantry angle } \\
\text { Depth }\end{array}$}} & \multicolumn{2}{|c|}{$15^{\circ}$} & \multicolumn{2}{|c|}{$30^{\circ}$} & \multicolumn{2}{|c|}{$60^{\circ}$} \\
\hline & & $5 \mathrm{~mm}$ & $15 \mathrm{~mm}$ & $5 \mathrm{~mm}$ & $15 \mathrm{~mm}$ & $5 \mathrm{~mm}$ & $15 \mathrm{~mm}$ \\
\hline \multirow{15}{*}{ 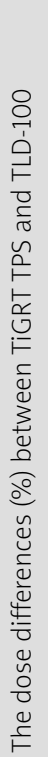 } & \multirow{5}{*}{$\begin{array}{l}\frac{\mathscr{U}}{\bar{N}} \\
\frac{\overline{0}}{\overline{0}} \\
\frac{0}{5} \\
\sim\end{array}$} & 17.55 & 2.66 & 16.65 & 9.19 & 20.86 & 17.89 \\
\hline & & 5.81 & -3.14 & 23.38 & 18.89 & 26.42 & 14.22 \\
\hline & & 11.24 & 7.88 & -2.57 & -4.11 & 25.78 & 11.38 \\
\hline & & 5.82 & 31.84 & 9.48 & 7.78 & 17.46 & 12.28 \\
\hline & & 18.07 & 4.69 & 32.39 & -1.66 & -10.27 & 14.46 \\
\hline & \multirow{4}{*}{ 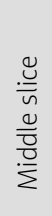 } & 13.99 & 18.75 & 15.09 & 12.97 & 19.77 & 11.27 \\
\hline & & 15.96 & 15.66 & 10.01 & 13.23 & 17.42 & 9.66 \\
\hline & & 5.28 & 21.63 & 7.68 & 6.75 & 15.66 & 16.76 \\
\hline & & 7.21 & -3.25 & 11.84 & 7.87 & 25.44 & 18.28 \\
\hline & \multirow{6}{*}{ 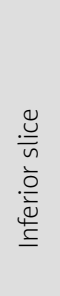 } & 20.8 & 7 & 9.35 & 13.11 & 24.93 & 18.85 \\
\hline & & 15.15 & 16.55 & 16.7 & 5.28 & 25.44 & 17.71 \\
\hline & & 10.09 & 23.75 & 8.54 & 21.22 & 15.81 & 19.31 \\
\hline & & 24.35 & 13.53 & 24.98 & 19.73 & 31.11 & 13.48 \\
\hline & & 10.98 & -2.32 & 10.08 & 13.56 & 28.73 & 18.04 \\
\hline & & 4.66 & 20.74 & -2.3 & 18.57 & 19.67 & 16.13 \\
\hline
\end{tabular}

The results showed that the dose differences between calculations and measurements for open and wedge fields at the depth of $5 \mathrm{~mm}$ for gantry angle of $60^{\circ}$ were greater than those of the other two gantry angles $\left(15^{\circ}\right.$ and $\left.30^{\circ}\right)$.

These findings demonstrated that dose differences between calculations and measurements for open and wedge fields at a depth of $15 \mathrm{~mm}$ for the gantry angle of $15^{\circ}$ were greater than those of the other two gantry angles $\left(30^{\circ}\right.$ and $\left.60^{\circ}\right)$. Furthermore, as the histograms in Fig. 3 and 4 show, for most points, that the TPS overestimates doses compared to the TLD measurements. Finally, the confidence limit values were obtained for the open and wedge fields in various gantry angles (Table 3).

\section{Discussion}

Most modern radiotherapy TPSs are able to accurately calculate doses in the high dose-small dose gradient region, while in most cases, doses calculated for high 

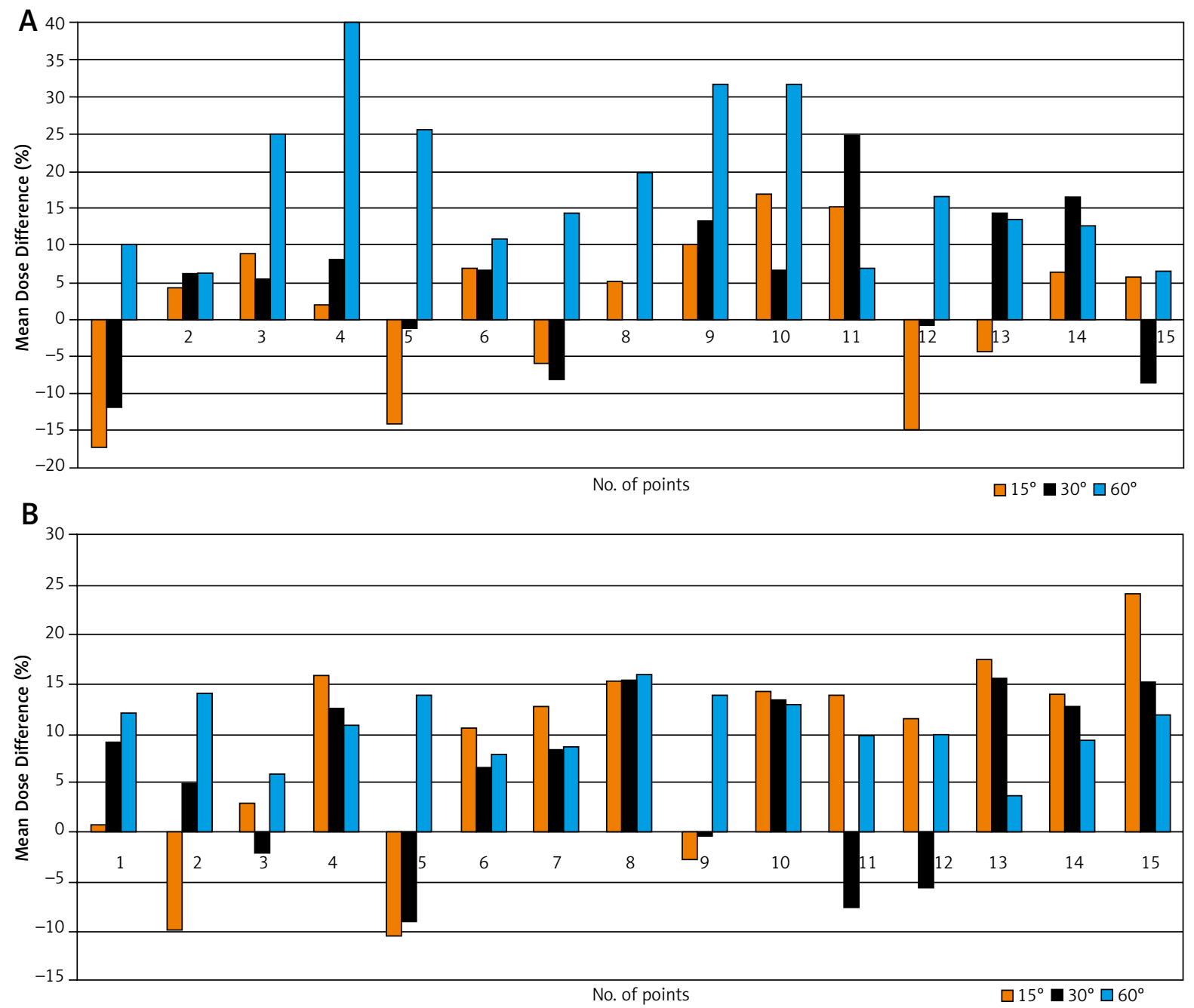

Fig. 3. Dose differences (\%) between the calculated doses by TiGRT TPS and the measured doses by TLD-100 thermoluminescent dosimeter for the open field with gantry angles of $15^{\circ}, 30^{\circ}$, and $60^{\circ}$ at depths of $5 \mathrm{~mm}(\mathrm{~A})$ and $15 \mathrm{~mm}$ (B), respectively

dose-large dose gradient regions such as the build-up region are inaccurate. This is recognised by the protocols for TPS commissioning and quality assurance, which suggest a tolerance limit of up to $20 \%$ in the high dose-large dose gradient region of the photon beam between the measurements and TPS calculations [33-35].

In the present study, the accuracy of dose calculations in the build-up region of the tangential field of the breast for TiGRT TPS was evaluated using TLD. Furthermore, one

Table 3. Confidence limit values obtained for the open and wedge fields in gantry angles of $15^{\circ}, 30^{\circ}$, and $60^{\circ}$

\begin{tabular}{lllll} 
& \multicolumn{4}{l}{ Confidence limit $(\Delta)$} \\
\cline { 2 - 5 } & Depth & $15^{\circ}$ & $30^{\circ}$ & $60^{\circ}$ \\
$\begin{array}{l}\text { Open } \\
\text { field }\end{array}$ & $5 \mathrm{~mm}$ & 17.68 & 19.97 & 34.62 \\
& $15 \mathrm{~mm}$ & 24.01 & 19.07 & 15.74 \\
$\begin{array}{l}\text { Wedge } \\
\text { field }\end{array}$ & $5 \mathrm{~mm}$ & 21.67 & 26.80 & 34.87 \\
& $15 \mathrm{~mm}$ & 27.92 & 22.04 & 20.03
\end{tabular}

of the strengths of this study over most other studies on dose calculation accuracy in the build-up region is the investigation of the effect of various gantry angles as well as the effect of wedge on TPS dose calculation accuracy at depths of 5 and $15 \mathrm{~mm}$ (build-up region).

As mentioned above, we used the TLD chips to measure the dose because this dosimetry method has been used in other studies of dose calculation accuracy in the build-up region. For example, Mutic and Low [36] used TLD and parallel-plate ionisation chambers to show that TomoTherapy TPS underestimates doses to the surface and near the surface by approximately $15 \%$. Budanec et al. [37] showed agreement within $1.5 \%$ between percentage depth doses measured by TLD and those calculated using the Monte Carlo method in the build-up region.

The results of this study showed that, for most points, the TPS overestimated doses compared to TLD measurements. Therefore, it is notable that delivering an insufficient dose in the surface build-up region, particularly when the tumour has spread to the skin, may cause tumour recurrence and should not rely on data obtained from the 


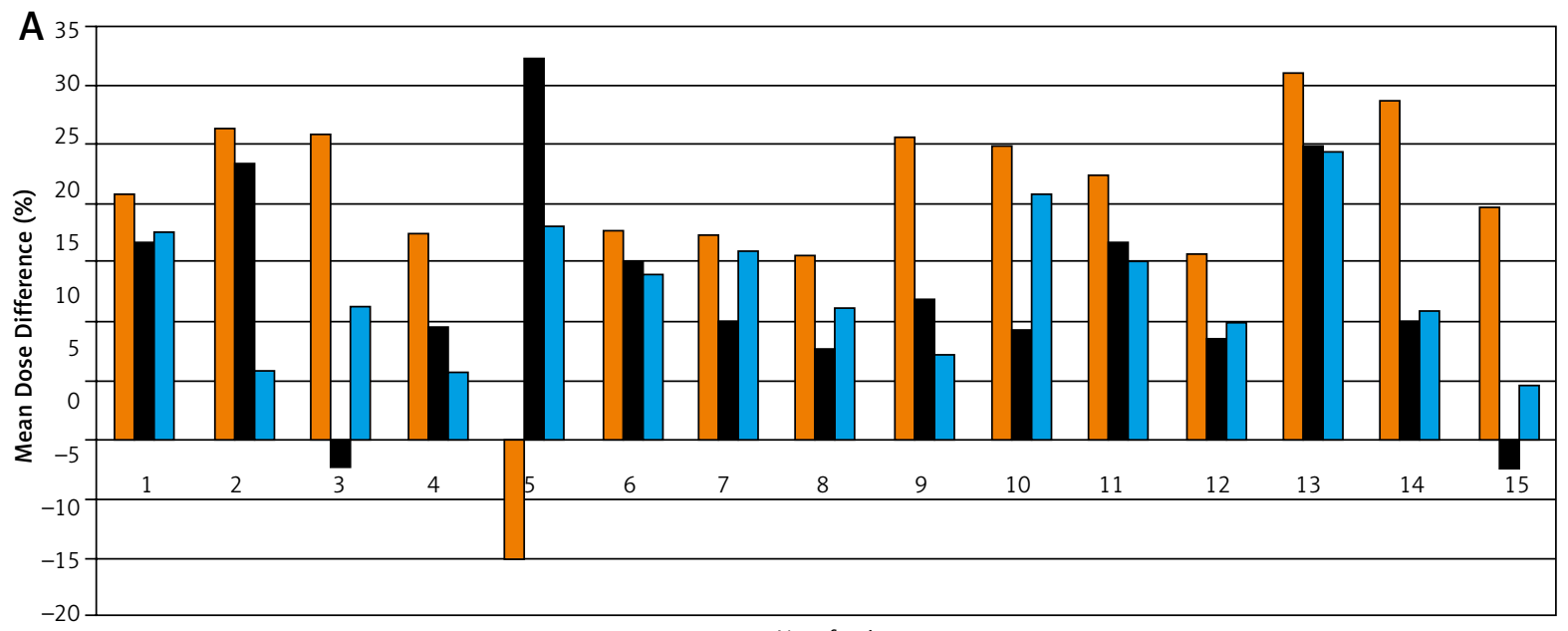

B

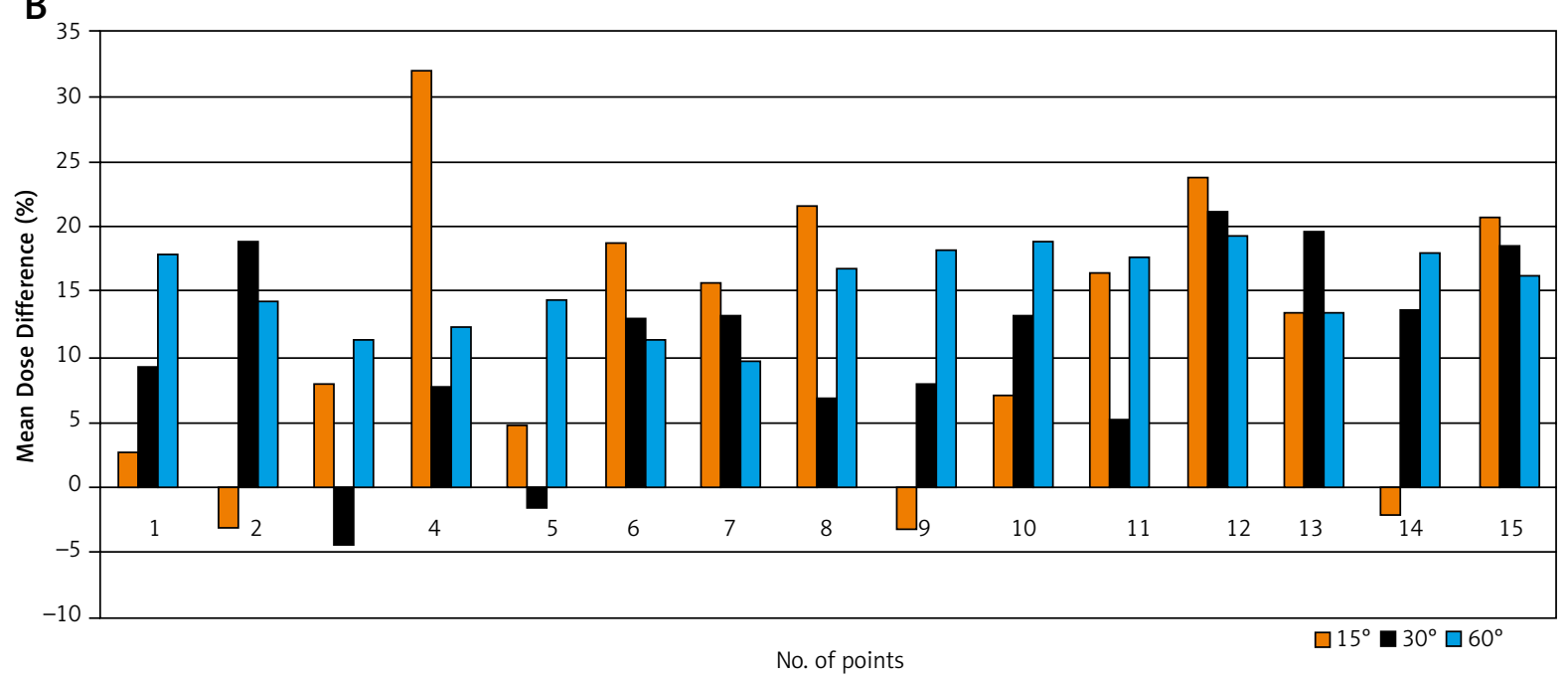

Fig. 4. Dose differences (\%) between the calculated doses by TiGRT TPS and the measured doses by TLD-100 thermoluminescent dosimeter for the wedge field with gantry angles of $15^{\circ}, 30^{\circ}$, and $60^{\circ}$ at depths of $5 \mathrm{~mm}(\mathrm{~A})$ and $15 \mathrm{~mm}(\mathrm{~B})$, respectively

TPS. This dose overestimation may be due to inaccurate modelling of the dose contributions from contaminated electrons and secondary scatter photons derived from the accelerator head. Our results were consistent with those of other studies. Chung et al. [38] reported that the TPS algorithm of Pinnacle and Corvus overestimated surface dose on a semi-cylindrical phantom. Oinam and Singh [36] concluded that the PBC algorithms of Eclipse TPS overestimated the doses compared to those measured by TLD in the high-dose build-up region. In addition, Farhood et al. [32] evaluated the dose calculation accuracy in the build-up region for TiGRT and prowess panther TPSs and showed for each of the three gantry angles $\left(15^{\circ}, 30^{\circ}\right.$, and $60^{\circ}$ ) that TiGRT TPS overestimated dose compared to the TLD-100 measurement of dose at most depths. However, our results were inconsistent with those of some other studies. Mutic and Low [39] and Chakarova et al. [17] showed that the Peacock and Eclipse TPS underestimated doses to and near the surface, while our results showed that the TiGRT TPS overestimated the doses.

For the open field, the confidence limit values at the depth of $5 \mathrm{~mm}$ for gantry angles of $15^{\circ}$ and $30^{\circ}$ were within the tol- erance limit value (20 for complex geometry in the build-up region), while for gantry angles of $60^{\circ}$ the confidence limit value was not within the tolerance limit. The confidence limit values for gantry angles of $30^{\circ}$ and $60^{\circ}$ at the depth of $15 \mathrm{~mm}$ were within the tolerance limit. In the assessment of the effect of gantry angle on TPS dose calculation accuracy in the open field in the build-up region at the depth of $5 \mathrm{~mm}$, the confidence limit values increased with increasing gantry angle; in contrast, at the depth of $15 \mathrm{~mm}$ the confidence limit values decreased with increasing gantry angle (Table 3).

For the wedge field, the confidence limit values at the depths of 5 and $15 \mathrm{~mm}$ for all gantry angles were not within the tolerance limit value; the tolerance limit value of 20 was assigned for the more complex geometry in the buildup region. An assessment of the effect of gantry angle on TPS dose calculation accuracy for the wedge field in the build-up region revealed similar results to those of the open field (Table 3). Farhood et al. [32] showed that the confidence limit values for TiGRT TPS in gantry angles of $15^{\circ}$ and $45^{\circ}$ were within the tolerance limit, while those for the gantry angle of $60^{\circ}$ were not within the tolerance limit. The results of our study are consistent with their results. 
It should be noted that the agreement and disagreement of our results with other studies may be due to differences in algorithm and TPS types used.

As a result, it should be noted that an excessive increase of build-up dose may cause severe reactions of the skin and under dose of underlying tissues [40]. Given that TiGRT TPS overestimated doses compared to TLD measurements, it is suggested that assessments of the dose distribution in build-up regions should not entirely rely on TPS calculations.

In conclusion, an accurate calculation of the dose distribution in the build-up region is essential to ensure adequate dose coverage of the target volume and prevent skin complications. The results of the present study showed that, for most points, TiGRT TPS overestimated doses compared to TLD measurements. Overall, the confidence limit values for the wedge field were greater than those for the open field. Our findings suggested that assessments of the dose distribution in large-dose gradient regions should not entirely rely on TPS calculations.

The authors would like to thank the Mashhad University of Medical Sciences (Mashhad, Iran) for financially supporting the present work.

The authors declare no conflicts of interest.

This work was financially supported by the office of vice president for research of Mashhad University of Medical Sciences.

\section{References}

1. Farhood B, Mahdavi SR, Emranpour MH, Mohammadi Asl K, Nekoui N, Knaup C. Skin Reaction in Radiation Therapy for Breast Cancer. Iran J Med Phys 2014; 11: 316-21.

2. Darby S, McGale P, Correa C, et al. Effect of radiotherapy after breast-conserving surgery on 10-year recurrence and 15-year breast cancer death: meta-analysis of individual patient data for 10,801 women in 17 randomised trials. Lancet 2011; 378: 1707-16.

3. Santiago RJ, Wu L, Harris E, Fox K, Schultz D, Glick J, Solin LJ. Fifteen-year results of breast-conserving surgery and definitive irradiation for Stage I and II breast carcinoma: the University of Pennsylvania experience. Int J Radiat Oncol Biol Phys 2004; 58: 233-40.

4. Veronesi U, Cascinelli N, Mariani L, Greco M, Saccozzi R, Luini A, Aguilar M, Marubini E. Twenty-year follow-up of a randomized study comparing breast-conserving surgery with radical mastectomy for early breast cancer. N Engl J Med 2002; 347: 1227-32.

5. Fisher B, Anderson S, Bryant J, Margolese RG, Deutsch M, Fisher ER, Jeong JH, Wolmark N. Twenty-year follow-up of a randomized trial comparing total mastectomy, lumpectomy, and lumpectomy plus irradiation for the treatment of invasive breast cancer. N Engl J Med 2002; 347: 1233-41.

6. Evans P, Hansen V, Mayles W, Swindell W, Torr M, Yarnold JR. Design of compensators for breast radiotherapy using electronic portal imaging. Radiother Oncol 1995; 37: 43-54.

7. Mussari S, Della Sala WS, Busana L, Vanoni V, Eccher C, Zani B, Menegotti L, Tomio L. Full-dose intraoperative radiotherapy with electrons in breast cancer. Strahlenther Onkol 2006; 182: 589-95.

8. Semrau S, Gerber B, Reimer T, Klautke G, Fietkau R. Concurrent radiotherapy and taxane chemotherapy in patients with locoregional recurrence of breast cancer. Strahlenther Onkol 2006; 182: 596-603.

9. Stranzl H, Ofner P, Peintinger F. Postoperative irradiation in breast cancer patients with one to three positive axillary lymph nodes. Strahlenther Onkol 2006; 182: 583-8.
10. Thilmann C, Zabel A, Kuhn S, Bendl R, Rhein B, Wannenmacher $M$, Debus J. Inversely planned intensity modulated radiotherapy for irradiation of a woman with breast cancer and funnel chest. Strahlenther Onkol 2002; 178: 637-43.

11. VanAken M, Breneman J, Elson HR, Foster AE, Lukes SJ, Little R. Incorporation of patient immobilization, tissue compensation and matchline junction technique for three-field breast treatment. Med Dosim 1988; 13: 131-5.

12. Venables K, Winfield EA, Aird EG, Hoskin PJ. Three-dimensional distribution of radiation within the breast: an intercomparison of departments participating in the START trial of breast radiotherapy fractionation. Int J Radiat Oncol Biol Phys 2003; 55: 271-9.

13. Parsai El, Shvydka D, Pearson D, Gopalakrishnan M, Feldmeier JJ. Surface and build-up region dose analysis for clinical radiotherapy photon beams. Appl Radiat Isot 2008; 66: 1438-42.

14. Panettieri V, Barsoum P, Westermark M, Brualla L, Lax I. AAA and PBC calculation accuracy in the surface build-up region in tangential beam treatments. Phantom and breast case study with the Monte Carlo code PENELOPE. Radiother Oncol 2009; 93: 94-101.

15. Akino Y, Das IJ, Bartlett GK, Zhang H, Thompson E, Zook JE. Evaluation of superficial dosimetry between treatment planning system and measurement for several breast cancer treatment techniques. Med Phys 2013; 40: 011714.

16. Nakano M, Hill RF, Whitaker M, Kim JH, Kuncic Z. A study of surface dosimetry for breast cancer radiotherapy treatments using Gafchromic EBT2 film. J Appl Clin Med Phys 2012; 13: 3727.

17. Chakarova R, Gustafsson M, Bäck A, et al. Superficial dose distribution in breast for tangential radiation treatment, Monte Carlo evaluation of Eclipse algorithms in case of phantom and patient geometries. Radiother Oncol 2012; 102: 102-7.

18. Kim S, Liu CR, Zhu TC, et al. Photon beam skin dose analyses for different clinical setups. Med phys 1998; 25: 860-6.

19. Biggs P, Russell M. An investigation into the presence of secondary electrons in megavoltage photon beams (radiotherapy application). Phys Med Biol 1983; 28: 1033-43.

20. Beauvais H, Bridier A, Dutreix A. Characteristics of contamination electrons in high energy photon beams. Radiother Oncol 1993; 29: 308-16.

21. Li Z, Klein EE. Surface and peripheral doses of dynamic and physical wedges. International J Radiat Oncol Biol Phys 1997; 37: 921-5.

22. Jackson $W$. Surface effects of high-energy $X$ rays at oblique incidence. Br J Radiol 1971; 44: 109-15.

23. Orton CG, Seibert JB. Surface effects of high-energy $X$ rays at oblique incidence. Br J Radiol 1971; 44: 895-6.

24. Khan FM. The physics of radiation therapy. $3^{\text {rd }}$ ed. Lippincott/Williams \& Wilkins, Philadelphila 2003.

25. Hounsell AR, Wilkinson JM. Electron contamination and build-up doses in conformal radiotherapy fields. Phys Med Biol 1999; 44: 43-55.

26. Sjögren R, Karlsson M. Electron contamination in clinical high energy photon beams. Med Phys 1996; 23: 1873-81.

27. Yang J, Li J, Qin L, Xiong W, Ma CM. Modelling of electron contamination in clinical photon beams for Monte Carlo dose calculation. Phys Med Biol 2004; 49: 2657-73.

28. Zhu TC, Palta JR. Electron contamination in 8 and $18 \mathrm{MV}$ photon beams. Med Phys 1998; 25: 12-9.

29. Lee CHM, Chan KKD. Electron contamination from the lead cutout used in kilovoltage radiotherapy. Phys Med Biol 2000; 45: 1-8.

30. Chow JC, Jiang R, Leung MK. Dosimetry of oblique tangential photon beams calculated by superposition/convolution algorithms: a Monte Carlo evaluation. J Appl Clin Med Phys 2010; 12: 3424.

31. Polednik D-IM, Madyan YA, Schneider F, et al.; Breast Cancer Working Group (German Cancer Association). Evaluation of calculation algorithms implemented in different commercial planning systems on an anthropomorphic breast phantom using film dosimetry. Strahlenther Onkol 2007; 183: 667-72.

32. Farhood B, Bahreyni Toossi M T, Ghorbani M, et al. Assessment the accuracy of dose calculation in build-up region for two radiotherapy treatment planning systems. J Cancer Res Ther 2017 (In press). Available: http://www.cancerjournal.net/preprintarticle. asp?id=176421; type $=0$. 
33. Andreo P, Cramb J, Fraass B, Shragge PC. Commissioning and quality assurance of computerized planning systems for radiation treatment of cancer. International Atomic Energy Agency technical report series 430 (TRS 430) 2004.

34. TECDOC I. 1540: Specification and acceptance testing of radiotherapy treatment planning systems. Vienna, International Atomic Energy Agency 2007.

35. Fraass B, Doppke K, Hunt M, Kutcher G, Starkschall G, Stern R, Van Dyke J. American Association of Physicists in Medicine Radiation Therapy Committee Task Group 53: quality assurance for clinical radiotherapy treatment planning. Med Phys 1998; 25: 1773-829.

36. Oinam AS, Singh L. Verification of IMRT dose calculations using AAA and PBC algorithms in dose buildup regions. J Appl Clin Med Phys 2010; 11: 3351.

37. Budanec M, Knežević Ž, Bokulić T, Mrcela I, Vrtar M, Vekić B, KusićZ. Comparison of doses calculated by the Monte Carlo method and measured by LiF TLD in the buildup region for a 60 Co photon beam. Appl Radiat Isot 2008; 66: 1925-1929.

38. Chung H, Jin H, Dempsey JF, Palta J, Suh TS, Kim S. Evaluation of surface and build-up region dose for intensity-modulated radiation therapy in head and neck cancer. Med Phys 2005; 32: 26822689.

39. Mutic S, Low DA. Superficial doses from serial tomotherapy delivery. Med Phys 2000; 27: 163-5.

40. Farhood B, Toossi MT, Ghatei N, Mohamadian N, Mozaffari A, Knaup C. A comparison between skin dose of breast cancer patients at the breast region, measured by thermoluminescent dosimeter in the presence and absence of bolus. J Cancer Res Ther 2017 (In press). Avelable: http://www.cancerjournal.net/preprintarticle.asp?id=188429; type $=0$.

\section{Address for correspondence}

\section{Mohammad Taghi Bahreyni Toossi}

Department of Medical Physics

Faculty of Medicine

Mashhad University of Medical Sciences

Pardis-e-Daneshgah, Vakil Abad Boulevard

Mashhad, Iran

e-mail: bahreynimt@mums.ac.ir

Submitted: 9.11 .2015

Accepted: $\quad 2.06 .2017$ 